\title{
SOME REMARKS ON PROJECTIVE STIEFEL MANIFOLDS, IMMERSIONS OF PROJECTIVE SPACES AND SPHERES
}

\author{
LARRY SMITH
}

\begin{abstract}
Let $M^{m}$ be a closed smooth manifold, $M \stackrel{\Phi}{\rightarrow} \mathbf{R}^{2 m}$ an immersion and $\tilde{M}^{m} \downarrow^{\pi} M^{m}$ a double covering. For $m$ odd we show that the normal bundle $\tilde{\nu} \downarrow \tilde{M}$ of the immersion $\tilde{M} \downarrow / M \leftrightarrow \mathbf{R}^{2 m}$ is independent of $\varphi$ and applying this to $M=$ $\mathbf{R} P(m)$ reobtain the result of E. H. Brown that a symmetric immersion $S^{m} \rightarrow R^{2 m}$ is regularly homotopic to an embedding iff $m=2^{p}-1$.
\end{abstract}

The theme of the Topologie Oberseminar during the SS 1978 was immersions and embeddings of manifolds. In the course of studying Whitney's classic papers on the subject a number of interesting problems arose. In particular, we spent quite some time trying to understand the Whitney invariant of $M^{m} \bullet \rightarrow R^{2 m}$. A particularly interesting question in this respect arises when one has a finite covering $\tilde{M}^{m} \downarrow^{\pi} M^{m}$ of closed manifolds, an immersion $M^{m} \vartheta^{\varphi} \rightarrow R^{2 m}$ with Whitney invariant $I_{\varphi}$, and one asks for a formula for the Whitney invariant of the composite immersion $\tilde{\varphi}$ : $\tilde{M}^{m} \downarrow^{\pi} M^{m Q^{\varphi}} \rightarrow \mathbf{R}^{2 m}$. Whitney's definition of $I_{\varphi}$ requires that the immersion be put in general position, and one then counts up the number of double points, with attention to orientation when $m$ is even, or simply takes the residue class mod 2 when $m$ is odd. The requirement of general position behaves very badly upon passing to a covering manifold. However in the even dimensional case $I_{\varphi}$ is one half the Euler class of the normal bundle, and it will simply be multiplied by the number of sheets in the covering upon passing to a covering manifold. The interesting case is $m$ odd. In this connection a theorem of E. H. Brown is quite striking: For any immersion $\mathbf{R} P(m) \stackrel{\stackrel{\varphi}{p}^{\rightarrow}}{\rightarrow} \mathbf{R}^{2 m}, m$ odd, the Whitney invariant of the composite immersion $\tilde{\varphi}: S^{m} \downarrow^{\pi} \mathbf{R} P(m) \bullet^{\varphi} \rightarrow \mathbf{R}^{2 m}$ is 0 iff $m=2^{p}-1$. This shows that $I_{\tilde{\varphi}}$ depends on more than just $I_{\varphi}$. This note is my "explanation" of Brown's theorem in terms of the Smale invariant as seen by a homotopy theorist.

After submission of this paper, the paper [0] of John Berrick appeared in the Proc. Cambridge Philos. Soc., and among other things, the theorem of Brown is reproved there along essentially the same lines as here. The theory developed by Berrick is more encompasing than our $\$ 2$ but does not seem as conceptual as the direct use of the projective Stiefel manifold and the universal property of the bundles over it expressed in Proposition 1.2 below.

Where appropriate, proofs that are easy modifications of arguments in [0] have been revised out.

Received by the editors August 17, 1979 and, in revised form, December 17, 1979 and February 18, 1980; presented in the Göttingen Topologie Oberseminar in May 1978.

1980 Mathematics Subject Classification. Primary 57R42. 
1. The projective Stiefel manifolds. Let $V_{k}\left(\mathbf{R}^{n}\right)$ denote the Stiefel manifold of $k$ frames in $\mathbf{R}^{n}$. There is the free involution

$$
T: V_{k}\left(\mathbf{R}^{n}\right) \rightarrow V_{k}\left(\mathbf{R}^{n}\right)
$$

given by

$$
T\left[v_{1}, \ldots, v_{k}\right]=\left[-v_{1}, \ldots,-v_{k}\right] .
$$

The orbit space of this involution is called the projective Stiefel manifold, and is denoted by $P V_{k}\left(\mathbf{R}^{n}\right)$. (See for example [4].) There is a canonical $d=n-k$ dimensional vector bundle $\xi \downarrow P V_{k}\left(\mathbf{R}^{n}\right)$ with total space

$$
E(\xi)=\left\{(x,\langle v\rangle) \in \mathbf{R}^{n} \times P V_{k}\left(\mathbf{R}^{n}\right), x \perp \operatorname{span}[v]\right\}
$$

and projection

$$
E(\xi) \downarrow P V_{k}\left(\mathbf{R}^{n}\right):(x,\langle v\rangle) \mapsto\langle v\rangle
$$

where $[v] \in V_{k}\left(\mathbf{R}^{n}\right)$ has orbit $\langle v\rangle \in P V_{k}\left(\mathbf{R}^{n}\right)$. We denote by $\lambda \downarrow P V_{k}\left(\mathbf{R}^{n}\right)$ the canonical line bundle associated to the double covering $V_{k}\left(\mathbf{R}^{n}\right) \downarrow P V_{k}\left(\mathbf{R}^{n}\right)$.

Proposition 1.1. $k \lambda \oplus \xi \simeq \mathbf{R}^{n} \downarrow P V_{k}\left(\mathbf{R}^{n}\right)$.

Proof. The total space $E(k \lambda \oplus \xi)$ has the following description

$$
\begin{array}{r}
E(k \lambda \oplus \xi)=\left\{(y, x,[v]) \in \mathbf{R}^{k} \times \mathbf{R}^{n} \times V_{k}\left(\mathbf{R}^{n}\right) \mid x \perp \operatorname{span}[v],\right. \\
(y, x, T[v]) \perp(-y, x,[v])\} .
\end{array}
$$

If $[y, x,[v]]$ denotes the equivalence class of $(y, x,[v]) \in \mathbf{R}^{k} \times \mathbf{R}^{n} \times V_{k}\left(\mathbf{R}^{n}\right)$ in $E(k \lambda \oplus \xi)$ then

$$
E(k \lambda \oplus \xi) \rightarrow \mathbf{R}^{n}:[y, x[v]] \mapsto x+\sum_{i=1}^{k} y_{i} v_{i}
$$

is a linear isomorphism on fibres, and hence a bundle trivialization.

Example $k=1$. Then $P V_{1}\left(\mathbf{R}^{n}\right)=\mathbf{R} P(n-1)$, the bundle $\lambda$ corresponds to the Hopf bundle, and the bundle $\xi$ to $\tau \otimes \lambda$ where $\tau \downarrow \mathbf{R P}(n-1)$ is the tangent bundle. The usual isomorphism

$$
\tau \oplus \mathbf{R} \simeq n \lambda \downarrow \mathbf{R} P(n-1)
$$

when tensored with $\lambda$ yields (1.1).

The isomorphism $k \lambda \oplus \xi \simeq \mathbf{R}^{n} \downarrow P V_{k}\left(\mathbf{R}^{n}\right)$ is in an appropriate sense universal. Specifically

Proposition 1.2. Let $X$ be a finite complex, $\zeta \downarrow X$ a line bundle, $\nu \downarrow X$ a d plane bundle and $k \zeta \oplus \nu \stackrel{\oplus}{\rightarrow} \mathbf{R}^{n} \downarrow X, n-k=d$, a bundle isomorphism. Then there exists $a$ map

$$
f: X \rightarrow P V_{k}\left(\mathbf{R}^{n}\right)
$$

such that $f^{*} \lambda=\zeta, f^{*} \xi=\nu \downarrow X$ and the isomorphism of (1.1) pulls back to the given isomorphism $\varphi$. 
Proof. Let $\tilde{X} \downarrow X$ be the double cover associated to the line bundle $\zeta$. View the trivialization $\varphi$ as a map

$$
E(k \zeta \oplus \nu) \rightarrow \mathbf{R}^{n}
$$

that on each fibre is a linear isomorphism. The composition

$$
\mathbf{R}^{k} \times \tilde{X} \hookrightarrow E\left(\mathbf{R}^{k} \oplus \pi^{*} \nu \downarrow \tilde{X}\right) \stackrel{\pi}{\rightarrow} E(k \zeta \oplus \nu \downarrow X) \stackrel{\varphi}{\rightarrow} \mathbf{R}^{n}
$$

then induces a map

$$
g: \mathbf{R}^{k} \times \tilde{X} \rightarrow \mathbf{R}^{n}
$$

with the properties

(1) $\forall x \in X, g(-, x): \mathbf{R}^{k} \rightarrow \mathbf{R}^{n}$ is a linear injection,

(2) $g(-u, \tilde{x})=g(u, t \tilde{x})$ where $t: \tilde{X} \rightarrow \tilde{X}$ is the covering transformation.

The adjoint of $g$

$$
\tilde{f}: \tilde{X} \rightarrow V_{k}\left(\mathbf{R}^{n}\right)
$$

is then an equivariant map, that is

$$
\tilde{f}(T x)=T f(x), \quad x \in X .
$$

There is therefore induced a map

$$
f: X \rightarrow P V_{k}\left(\mathbf{R}^{n}\right)
$$

and it follows more or less from the definition that $f^{*} \lambda \simeq \zeta, f^{*} \xi \simeq \nu$ and that $f$ pulls the isomorphism of (1.1) back to $\varphi$.

2. Applications to immersions of projective spaces.

Proposition 2.1. (Compare [7, 2.3].) If $\varphi: \mathbf{R} P(m) \leftrightarrow \mathbf{R}^{m+k}$ is an immersion with normal bundle $\nu$, then there exists a map $f_{\varphi}: \mathbf{R} P(m) \rightarrow P V_{m+1}\left(\mathbf{R}^{m+k+1}\right)$ such that $f_{\varphi}^{*} \lambda=\gamma, f_{\varphi} \xi=\nu \downarrow \mathbf{R} P(m)$ where $\gamma \downarrow \mathbf{R} P(m)$ is the Hopf bundle.

Proof. One has bundle isomorphisms

$$
\begin{aligned}
& \tau \oplus \nu_{\varphi} \simeq \mathbf{R}^{m+k} \downarrow \mathbf{R} P(m), \\
& \mathbf{R} \oplus \tau \simeq(m+1) \gamma \downarrow \mathbf{R} P(m),
\end{aligned}
$$

which together give

$$
(m+1) \gamma \oplus \nu_{\varphi} \simeq \mathbf{R}^{m+k+1} \downarrow \mathbf{R} P(m)
$$

to which (1.2) applies.

COROLlaRY 2.2. If $\varphi: \mathbf{R} P(m) \stackrel{\natural}{\rightarrow} \mathbf{R}^{m+k+1}$ is an immersion, then $\varphi$ induces an axial map

$$
A_{\varphi}: \mathbf{R} P(m) \times \mathbf{R} P(m) \rightarrow \mathbf{R} P(m+k) .
$$

Proof. Let

$$
f_{\varphi}: \mathbf{R} P(m) \rightarrow P V_{m+1}\left(\mathbf{R}^{m+k+1}\right)
$$

classify $\nu_{\varphi}$. The "adjoint" of $f$ is the required axial map $A_{\varphi}$. 
Proposition 2.3. Suppose $g: \operatorname{RP}(m) \rightarrow P V_{m+1}\left(\mathbf{R}^{m+k+1}\right)$ so that $g^{*} \lambda=\gamma$. Then $g$ induces an immersion $\varphi_{g}: \mathbf{R} P(m) \rightarrow \mathbf{R}^{m+k+1}$ with normal bundle $g^{*} \xi \downarrow \mathbf{R} P(m)$.

Proof. From the universal example we get by pullback

$$
(m+1) \gamma \oplus g^{*} \xi \simeq \mathbf{R}^{m+k+1} \downarrow \mathbf{R} P(m)
$$

and as usual we have

$$
(m+1) \gamma \simeq \mathbf{R} \oplus \tau \downarrow \mathbf{R} P(m) .
$$

Combining gives

$$
\mathbf{R} \oplus \tau \oplus g^{*} \xi \simeq \mathbf{R}^{m+k+1} \downarrow \mathbf{R} P(m)
$$

and stability allows us to conclude

$$
\tau \oplus g^{*} \xi \simeq \mathbf{R}^{m+k} \downarrow \mathbf{R} P(m)
$$

and the result follows by applying Hirsch's theorem [5].

REMARK. Suppose that

$$
f: \mathbf{R}^{m+1} \times \mathbf{R}^{m+1} \rightarrow \mathbf{R}^{m+k+1}
$$

is a nonsingular bilinear form. Ginsberg [3] proved that $f$ induces an immersion

$$
\varphi_{f}: \mathbf{R} P(m) \leftrightarrow \mathbf{R}^{m+k} .
$$

From our viewpoint this can be seen more explicitly as follows. The adjoint of $f$ induces a map

$$
\operatorname{ad} f: S^{m} \rightarrow V_{m+1}\left(\mathbf{R}^{m+k+1}\right)
$$

satisfying

$$
\operatorname{ad} f(-x)=T \text { ad } f(x)
$$

and hence inducing

$$
\overline{\operatorname{ad} f}: \mathbf{R} P(m) \rightarrow P V_{m+1}\left(\mathbf{R}^{m+k+1}\right)
$$

such that

$$
\overline{\operatorname{ad} f}^{*} \lambda=\gamma \downarrow \mathbf{R} P(m) .
$$

From (2.3) we thus obtain an immersion

$$
\varphi_{f}: \mathbf{R} P(m) \leftrightarrow \mathbf{R}^{m+k}
$$

with normal bundle $\overline{\operatorname{ad} f^{*}}(\xi)$. This normal bundle can be more precisely described as follows

$$
E\left(\nu_{\varphi_{j}}\right)=\left\{([x], w) \in \mathbf{R} P(m) \times \mathbf{R}^{m+k+1} \mid w \perp \text { ad } f(x)\left(\mathbf{R}^{m+1}\right)\right\} .
$$

\section{Symmetric immersions of spheres.}

Definition. An immersion $\psi: S^{m} \leftrightarrow \mathbf{R}^{m+k}$ is called symmetric if $\psi(-x)=\psi(x)$.

EXAMPLE. Suppose $f: \mathbf{R}^{m+1} \times \mathbf{R}^{m+1} \rightarrow \mathbf{R}^{m+k+1}$ is a symmetric nonsingular bilinear form. Then the map

$$
\psi_{f}: S^{m} \rightarrow S^{m+k}: x \mapsto \frac{f(x, x)}{\|f(x, x)\|}
$$


is a symmetric immersion. For example the form $(m, m)_{\mathbb{R}}$ induces a symmetric immersion $S^{m} \rightarrow \mathbf{R}^{2 m}$.

REMARK. A symmetric immersion $\psi: S^{m} \leftrightarrow \mathbf{R}^{m+k}$ induces an immersion $\psi$ : $\mathbf{R} P(m) \bullet \mathbf{R}^{m+k}$ and conversely.

PRoposition 3.1. An immersion $\psi: S^{m} \leftrightarrow \mathbf{R}^{m+k}$ is symmetric up to regular homotopy iff the Smale invariant $\sigma_{\psi} \in \pi_{m}\left(V_{m+1}\left(\mathbf{R}^{m+k+1}\right)\right)$ admits a skew representative.

Proof. An immersion $\varphi$ is symmetric exactly when it induces an immersion $\bar{\psi}$ : $\mathbf{R} P(m) \bullet \mathbf{R}^{m+k}$, and the result follows by simply applying the definitions (see [0, §2]).

REMARK. Taking the value of the homotopy groups of the Stiefel manifolds into account one sees that the Smale invariant is a global formulation of the Whitney invariant.

As an application of the forgoing discussion we reprove a theorem of $\mathrm{E}$. $\mathrm{H}$. Brown [1].

Proposition 3.2. Let $m$ be odd and $S^{m} \vartheta^{\psi} \rightarrow R^{2 m}$ a symmetric immersion. Then the Smale invariant $\sigma_{\psi} \in \pi_{m}\left(V_{m+1}\left(\mathbf{R}^{2 m+1}\right)\right)=Z / 2$ is nonzero iff $m=2^{p}-1$. In particular, the normal bundle $\nu_{\psi}$ is nontrivial, and hence isomorphic to $\tau \downarrow S^{m}$ iff $m=2^{p}-$ $1, p>3$.

Proof. In view of (3.1) it suffices to show that a skew map $S^{m} \rightarrow V_{m+1}\left(\mathbf{R}^{2 m+1}\right)$ is null homotopic iff $m \neq 2^{p}-1$.

To this end we make use of the following (for a proof see (3.3)).

FACT. The normal bundle $\nu \downarrow S^{m}$ of a symmetric immersion $S^{m} \stackrel{\psi}{\rightarrow} \mathbf{R}^{2 m}, m$ odd, does not depend on $\psi$.

In this way see that for $m \neq 1,3,7$, exactly one of the two classes in $\pi_{m}\left(V_{m+1}\left(\mathbf{R}^{2 m+1}\right)\right)=Z / 2$ is skew. It will suffice therefore to show that $0 \in$ $\pi_{m}\left(V_{m+1}\left(\mathbf{R}^{2 m+1}\right)\right)$ is skew iff $m \neq 2^{p}-1$. The following neat little argument I owe to the referee.

If $m+1 \neq 2^{p}$ then, as is well known, $\mathbf{R} P(m+1)$ immerses in $\mathbf{R}^{2 m}$. Therefore by Proposition 3.1 there is a skew element in $\pi_{m+1}\left(V_{m+2}\left(\mathbf{R}^{2 m+1}\right)\right)$. Since the projection map $V_{m+2}\left(\mathbf{R}^{2 m+1}\right) \downarrow V_{m+1}\left(\mathbf{R}^{2 m+1}\right)$ is equivariant, there is a skew element in $\pi_{m+1}\left(V_{m+1}\left(\mathbf{R}^{2 m+1}\right)\right)$, which upon restricting to a codimension 1 subsphere, represents $0 \in \pi_{m}\left(V_{m+1}\left(\mathbf{R}^{2 m+1}\right)\right)$ via a skew map.

It remains to show that when $m=2^{p}-1$, a skew map

$$
f: S^{m} \rightarrow V_{m+1}\left(\mathbf{R}^{2 m+1}\right)
$$

must be essential. Supposing the contrary, we can extend $f$ to ( $D^{m}$ is an $m$ disk)

$$
F: D^{m} \rightarrow V_{m+1}\left(\mathbf{R}^{2 m+1}\right)
$$

and define

$$
g: S^{m+1} \rightarrow V_{m+1}\left(\mathbf{R}^{2 m+1}\right)
$$


by

$$
g\left(x_{0}, \ldots, x_{m+1}\right)=\left\{\begin{array}{l}
F\left(x_{0}, \ldots, x_{m+1}\right), \quad x_{m+1}>0, \\
T F\left(-x_{0}, \ldots,-x_{m+1}\right), \quad x_{m+1}<0,
\end{array}\right.
$$

which because of the skewness of $\left.F\right|_{s^{m}}=f$ is well defined and skew. The unadjoint of $g$ yields an axial map

$$
\mathbf{R} P(m+1) \times \mathbf{R} P(m) \stackrel{A}{\rightarrow} \mathbf{R} P(2 m)
$$

and hence by Hopf (use that $A^{*} w_{1}(\gamma)^{2 m+1}=0$ )

$$
\left(\begin{array}{c}
2 m+1 \\
m
\end{array}\right) \equiv 0 \bmod 2 \text {. }
$$

But

$$
\left(\begin{array}{c}
2 m+1 \\
m
\end{array}\right)=\left(\begin{array}{c}
2^{p}+\cdots+1 \\
2^{p}
\end{array}\right) \equiv 1 \bmod 2
$$

which is a contradiction. Hence the original skew map $f$ must be essential as required.

The "Fact" used in the proof of (3.2) was pointed out to me by Ch. Okonek and is a special case of the following result, that is of interest in its own right.

Proposition 3.3. Let $\tilde{M} \downarrow^{\pi} M^{m}$ be a double covering and $M^{m} \Omega^{\phi} \rightarrow \mathbf{R}^{2 m}$ an immersion, $m$ odd. Then the normal bundle of the immersion $\tilde{M}^{m} \downarrow^{\pi} M^{m} \leftrightarrow \mathbf{R}^{2 m}$ is independent of $\varphi$.

Proof. Let $M^{m} \stackrel{\Phi_{i}}{\rightarrow} \mathbf{R}^{2 m}$ be immersions with normal bundles $\nu_{i} \downarrow M^{m}$, where $i=1,2$. Then (see for example [2]) there exists a stably trivial $m$-plane bundle $\xi \downarrow S^{m}$ such that

$$
\nu_{1} \simeq p^{*}\left(\nu_{2} \vee \xi\right)
$$

where

$$
p: M \rightarrow M \vee S^{m}
$$

is the map that pinches the boundary of a small disk in $M$ to a point. The composite immersions $\tilde{M}^{m} \downarrow M^{m_{\vartheta}} \stackrel{\varphi_{i}}{\leftrightarrow} \mathbf{R}^{2 m}$ have normal bundles $\tilde{\nu}_{i}=\pi^{*} \nu_{i} \downarrow \tilde{M}^{m}$, for $i=1$, 2. From the commutative diagram

$$
\begin{array}{ccc}
\tilde{M}^{m} & \stackrel{\tilde{p}}{\rightarrow} & \tilde{M}^{m} \vee S^{m} \\
\downarrow & & \\
M^{m} & \stackrel{p}{\rightarrow} & M^{m} \vee S^{m}
\end{array}
$$

where $\tilde{p}$ pinches the boundary of a small disk in $\tilde{M}^{m}$ to a point, and $h$ has degree 2, one sees that

$$
\tilde{\nu}_{1} \simeq \tilde{p}^{*}\left(\nu_{2} \vee h^{*} \xi\right)
$$

However since $m$ is odd

$$
\operatorname{ker}\left\{\pi _ { m } ( B S O ( m ) ) \rightarrow \pi _ { m } ( B S O ) \simeq \left\{\begin{array}{lr}
0, & m=1,3,7, \\
Z / 2, & \text { otherwise, }
\end{array}\right.\right.
$$

and hence $h^{*} \xi \simeq \mathbf{R}^{m}$. Therefore $\tilde{\nu}_{1} \simeq \tilde{\nu}_{2} \downarrow M^{m}$ as required. 
Remarks. (1) A symmetric immersion of

$$
\varphi: S^{m} \leftrightarrow \mathbf{R}^{2 m} \subset \mathbf{R}^{2 m+1}-0
$$

for any positive integer $m$ is given by the formula

$$
\begin{gathered}
\varphi\left(x_{0}, \ldots, x_{m}\right)=\left(z_{0}, \ldots, z_{2 m}\right), \\
z_{k}=\sum_{i+j=k} x_{i} x_{j}, \quad k=0, \ldots, 2 m .
\end{gathered}
$$

It would be very interesting to understand exactly why the Whitney invariant of this immersion is 1 when $m=2^{p}-1$ and 0 otherwise.

\section{REFERENCES}

0. A. J. Berrick, The Smale invariant of an immersed projective space, Proc. Cambridge Philos. Soc. 80 (1979), 401-411.

1. E. H. Brown, Jr., A remark concerning immersions of $S^{n}$ in $\mathbf{R}^{2 n}$, Quart. J. Math. Oxford Ser. 24 (1973), 559-560.

2. J. Dupont, On the homotopy invariance of the tangent bundle. I, Math. Scand. 26 (1970), 5-13.

3. M. Ginsburg, Some immersions of projective spaces in euclidean space, Topology 2 (1963), 69-72.

4. S. Gitler and D. Handel, Projective Stiefel manifolds. I, Topology 7 (1968), 39-46.

5. M. Hirsch, Immersions of manifolds, Trans. Amer. Math. Soc. 93 (1959), 242-276.

6. M. Kervaire, Sur l'invariant de Smale d'un plongement, Comment. Math. Helv. 34 (1960), $127-139$.

7. B. J. Sanderson, A non-immersion theorem for real projective space, Topology 2 (1963), 209-211.

8. B. J. Sanderson, Immersions and embedding of projective space, Proc. London Math. Soc. 16 (1964), 135-153.

9. S. Smale, The classification of immersions of spheres in euclidean space, Ann. of Math. 69 (1959), 327-344.

Mathematisches Institut Universträt Göttingen, Bunsenstrasse 3/5, 3400 Göttingen, West GermaNY 